\title{
On the Use of an Improved Artificial Fish Swarm Algorithm-Backpropagation Neural Network for Predicting Dam Deformation Behavior
}

\author{
Bo Dai $\mathbb{D}^{1,2,3}$ Hao Gu $\mathbb{D}^{2,4}$, Yantao Zhu $\mathbb{D}^{1,},{ }^{1,2,3}$ Siyu Chen $\mathbb{D}^{1,},{ }^{1,2,3}$ and E. Fernandez Rodriguez ${ }^{5}$ \\ ${ }^{1}$ State Key Laboratory of Hydrology-Water Resources and Hydraulic Engineering, Hohai University, Nanjing 210098, China \\ ${ }^{2}$ College of Water Conservancy and Hydropower Engineering, Hohai University, Nanjing 210098, China \\ ${ }^{3}$ National Engineering Research Center of Water Resources Efficient Utilization and Engineering Safety, Hohai University, \\ Nanjing 210098, China \\ ${ }^{4}$ College of Agricultural Engineering, Hohai University, Nanjing 210098, China \\ ${ }^{5}$ Technological Institute of Merida, Technological Avenue, Merida 97219, Mexico
}

Correspondence should be addressed to Hao Gu; ghao@hhu.edu.cn and Yantao Zhu; zyt50@163.com

Received 21 July 2020; Revised 17 September 2020; Accepted 12 October 2020; Published 28 October 2020

Academic Editor: Zong Woo Geem

Copyright $\odot 2020$ Bo Dai et al. This is an open access article distributed under the Creative Commons Attribution License, which permits unrestricted use, distribution, and reproduction in any medium, provided the original work is properly cited.

Dam behavior is difficult to predict due to its complexity. At the same time, dam deformation behavior is vital to dam systems. Developing a precise prediction model of dam deformation from prototype data is still challenging but determinant in the structural safety assessment. In this paper, an artificial neural network (ANN), trained by the improved artificial fish swarm algorithm (IAFSA) and backpropagation algorithm (BP), is proposed for predicting the dam deformation. Initially, crossover operator is embedded into AFSA, which aims to enhance the performance. In light of the influence mechanism of many factors on dam deformation behavior, the hybrid (IAFSA and BP) model uses statistical input to obtain the optimal connection weights and threshold values of the neural network. The hybrid model integrates IAFSA's strong global searching ability and BP's strong local search ability. To avoid overfitting the training set data, a validation set is adopted to check the generalization capability. Subsequently, the obtained optimal parameters are applied to predict the dam deformation behavior. The hybrid model's preciseness is verified against the radial displacements of a pendulum in a concrete arch dam and simulations of four models: statistical model, BP-ANN optimized by genetic algorithm (GA), particle swarm optimization (PSO), and AFSA. Results demonstrate that the proposed model outperforms other models and may provide alarms for safety control.

\section{Introduction}

Dam failures are generally catastrophic if the structure is breached [1]. Dam is a complex system, which comprises many interconnected components, such as the dam body, the spillway, the power plant, etc. Dam behavior is intrinsically difficult to predict due to the interactions between their components or between the given dam system and its environment. Through dam safety monitoring, prototype observations on dam behavior can be obtained. Among the various observations, the most important indicator is deformation [2]. Over the past years, researches have dealt relatively well with the dam deformation through models, based on prototype observations [3-6].

The prediction models, in the context of dam safety monitoring, offer a solution, by first creating knowledge from previous experiences and learning the model parameters, in order to predict the dam behavior from possible input data [7]. They have the attractiveness of being robust and effective with insufficient input information, noise, and uncertainty [7]. Notable data-based behavior models include the genetic algorithm (GA) $[8,9]$, particle swarm optimization (PSO) [10], differential evolution (DE) [11], gravitational search algorithm (GSA) [12], harmony search algorithm [13, 14], support vector machine (SVM) [15], 
dendritic neuron model (DNM) [16, 17], boosted regression trees $[18,19]$, extreme learning machine $[20,21]$, and artificial neural network (ANN) [22]. The artificial neural network (ANN) $[23,24]$ is useful for detecting nonlinear patterns and has been widely used in many different domains, including dam engineering [25-27].

To train the neural network in an efficient manner, tools such as backpropagation algorithm (BP) [28] are used in the data-based behavior models. The BP neural network [29] algorithm is a multi-layer feedforward network, trained according to error backpropagation algorithm, and it is one of the most widely applied neural network models. BP network can be used to learn and store a great deal of mapping relations of input-output model and requires no previous knowledge of the mathematical equation describing the mapping relationships. Its learning rule is to adopt the steepest descent method, where the weight value and threshold value of the network are regulated to achieve the minimum sum-of-squared error between the desired and computed output [30].

Despite the fact that the use of BP algorithm is justified [31] for most conditions, problems might appear in the solution of complex descent gradients due to inherent trapment of local rather than global minima [32]. In recent years, researchers have been investigating approaches to counteract this disadvantage, such as defining modified [33] or hybrid forms of BP algorithms. In particular, the hybrid form has gained fresh prominence in the dam deformation models with many arguing it leads to fasterconverging and better results, as opposed to the original BP algorithm [34].

This paper proposes the hybrid BP and artificial fish swarm algorithm (AFSA) model for understanding dam behavior. The AFSA [35] has been employed to optimize the swarm intelligence. The basic premise of the AFSA is to imitate the fish behaviors such as preying, swarming, and chasing, where the search of fish individuals leads to the global optimal solution [35]. This approach contributes to accurate results, high rate of convergence, flexibility, and tolerance to failure $[36,37]$. To enhance its optimization performance, a novel variant has been proposed, named improved artificial fish swarm algorithm, or IAFSA. In this crossover operator, the artificial fish acquire their parents' attributes, promoting the diversification and rising the likelihood of attaining the global optimal solution.

The rest of the paper is organized as follows. In Section 2.1, the statistical relations between loads and deformation behavior are briefly introduced. Section 2.2 deals with the concepts of improved artificial fish swarm algorithm (IAFSA). Artificial neural network (ANN) and backpropagation (BP) algorithm are introduced in Section 2.3 and Section 2.4. In Section 2.5, the overall structure of hybrid-learning algorithm, IAFSA-BP, is introduced for training the ANN. A dam case study is described in Section 3. In Section 4, the prediction accuracy and potential of the proposed algorithm are verified. And discussions and conclusions are given in Sections 4 and 5, respectively.

\section{Hybrid IAFSA-BP-ANN Model}

2.1. Statistical Relations between Loads and Deformation Behavior of Dam. In order to provide reliable structural responses, the mechanisms of the factors influencing the dam deformation behavior need to be investigated and reproduced in a simple but yet accurate way [38]. Statistical (also hydrostatic-season-time (HST) model) methods fit this criteria.

In this sense, a multi-period harmonic can be selected as the factor of dam temperature variation, once the hydration heat has been distributed and the quasi-steady temperature field has been reached in the dam body. The statistical relations between loads and the deformation behavior of the dam $(\delta)$ can be described as the sum of three terms: the temperature variation, the hydrostatic pressure variation, and other unexpected causes, such as time effects. It is [38]

$$
\begin{aligned}
\delta= & \delta_{H}+\delta_{T}+\delta_{\theta}=a_{0}+\sum_{i=1}^{m_{1}} a_{i} H^{i} \\
& +\left(\sum_{i=1}^{m_{2}} b_{1 i} \sin \frac{2 \pi i t}{365}+b_{2 i} \cos \frac{2 \pi i t}{365}\right)+c_{1} \theta+c_{2} \ln \theta
\end{aligned}
$$

where $\delta_{H}$ is the hydrostatic pressure component; $\delta_{T}$ is the temperature component; $\delta_{\theta}$ is the time effect component; $a_{0}$ is constant; $a_{i}, b_{1 i}, b_{2 i}, c_{1}$, and $c_{2}$ represent coefficients; $H$ is water load; $m_{1}$ is an integer dependent on the dam type ( 3 for gravity dam, 4 or 5 for arch dam); $i$ denotes the period (1 for annual cycle, 2 for half a cycle); $t$ is the cumulative number of days from the initial value to monitoring value; $\theta=t / 100$; and $m_{2}$ is usually taken as 1 or 2 . The effect of hydrostatic thrust, seasonal environment, and irreversible time-dependent conditions are regarded as inputs of the ANN.

\subsection{Improved Artificial Fish Swarm Algorithm}

2.2.1. Review of Artificial Fish Swarm Algorithm. In nature, an area occupied with large fish number is generally nutritious. The fish can discover the most nutritious area by intelligent behaviors, such as preying behavior, swarming behavior, following behavior, etc. [35]. The artificial fish swarm algorithm (AFSA) is an artificial intelligent algorithm based on the behavior of fish swarm. This algorithm is capable of reaching the global optimum by imitating the collective movement of artificial fish (AF) [36, 39]. The AFSA has attractive features, such as good robustness, global search ability, tolerance of parameter setting, and insensitiveness to initial values $[35,36]$.

The vision concept of the AF is illustrated in Figure 1. From the graph, the term Step is the step length and the Visual is the visual distance. The spatial coordinate of the AF is represented by $X=\left(x_{1}, x_{2}, x_{3}, \ldots, x_{n}\right)$, where $x_{i}$ is a potential solution. The food consistence of the AF in its current position is denoted by the objective function, $Y=f(X)$. The distance between adjacent AF individuals (ith and $j$ th) is expressed as $D_{\mathrm{ij}}=\left\|X_{i}-X_{j}\right\|$ and the crowd factor is $\Delta$. 


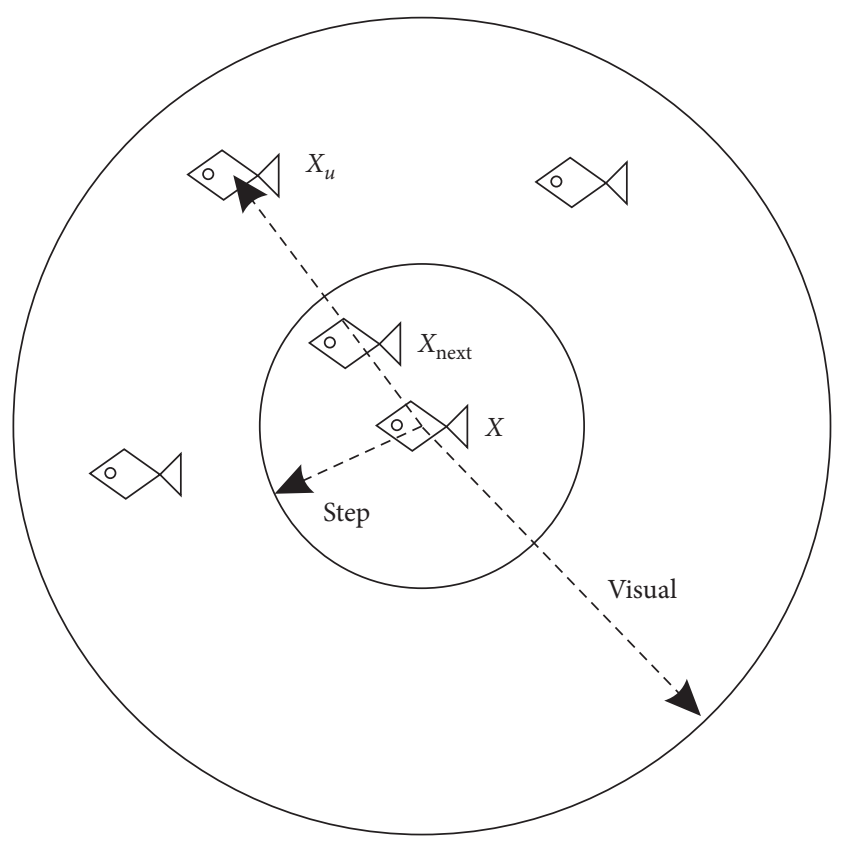

FIgURE 1: Vision concept of the artificial fish.

The fish behavior relies on inspecting the nearby region, up to where a behavior condition is met. Thus, if the AF is conditioned to step forward in a direction, it arrives to $X_{\text {next }}$; otherwise, it continues inspecting within its visual range.

The refreshed position can be described as

$$
\begin{aligned}
x_{v} & =x_{i}+\text { Visual } \times \text { Rand } i \in(0, n], \\
X_{\text {next }} & =\frac{X_{v}-X}{\left\|X_{v}-X\right\|} \times \text { Step } \times \text { Rand }
\end{aligned}
$$

where $X_{v}$ is a position within the vision; Rand are randomly generated numbers between zero and 1 ; and $n$ is the number of variables; others are the same as above.

The AF model contains four classical behaviors: preying behavior, swarming behavior, following behavior, and random behavior.

(1) Preying Behavior. Preying behavior is mainly considered as a kind of behavior of tending to more food. Within the context of optimization algorithm, it is an iterative way of moving to a more nutritious area, such as in the visual concept in the AF.

Let the current location of the AF be $X_{i}$, and a random position in the visual range be $X_{j}$. Then, take the maximum problem as an example (because the maximum problem can be transformed into minimum problem via the reciprocal of the objective function) and the position becomes

$$
X_{j}=X_{i}+\text { Visual } \times(2 \times \text { rand }-1),
$$

where other terms are the same as above.

Thus, if the objective criterion, $Y_{i}<Y_{j}$, is met, the AF steps forward in this direction; otherwise, select a new random location $X_{j}$, and execute the objective condition. If the condition is not satisfied, after a specified number of times, called the try_number, step randomly. In the preying behavior, a small try_number implies the AF swims randomly and thus diverges from the local extreme value field. The refreshed position is

$$
\left\{\begin{array}{l}
X_{i}(t+1)=X_{i}(t)+\frac{X_{j}(t)-X_{i}(t)}{\left\|X_{j}(t)-X_{i}(t)\right\|} \times \operatorname{Step} \times \operatorname{rand}() \quad Y_{i}<Y_{j}, \\
\text { Equation(4), } Y_{i} \geq Y_{j}
\end{array}\right.
$$

where the terms are the same as above.

(2) Swarming Behavior. The swarming behavior can be described, using two rules from Reynolds [40]:

To move to the center of the nearest companions as much as possible.

To avoid overcrowding, so the swarming ability of the artificial fish can be basically realized.

When travelling, the fish assemble in groups naturally to avoid danger and guarantee the existence of the colony. Let $X_{c}$ be the center of this gathering area

$$
X_{c}=\frac{1}{n} \sum_{i=1}^{n} X_{i} \text {, }
$$

where $n$ is the total fish population.

Let $n_{f}$ be the number of AF's companions in the neighborhood $\left(d_{i j}<\right.$ Visual). If $\left(Y_{c} / n_{f}\right)>\delta Y_{i}$, it means the AF's companion center has more food (higher fitness function value), with a small crowd factor; hence, the AF steps to the companion center; otherwise, the AF executes the preying behavior. The updated position conditions are

$\left\{\begin{array}{l}X_{i}(t+1)=X_{i}(t)+\frac{X_{c}(t)-X_{i}(t)}{\left\|X_{c}(t)-X_{i}(t)\right\|} \times \operatorname{Step} \times \operatorname{rand}() \frac{Y_{c}}{n_{f}}>\delta Y_{i}, \\ \text { equation (4), otherwise, }\end{array}\right.$

where the terms are the same as above.

(3) Following Behavior. The following behavior can be understood as moving towards the best neighbor companions. In the random behavior, the direction is not specified due to a lack of purpose.

Let $X_{i}$ be the AF current position, and the companion in the neighborhood $\left(d_{i j}<\right.$ Visual $)$ with the greatest food consistence $X_{j}$. If $\left(Y_{j} / n_{f}\right)>\delta Y_{i}$, the AF steps forward due to companion's higher food concentration (higher fitness function value) with spacious surrounding; otherwise, it follows the preying behavior. The conditions are

$$
\left\{\begin{array}{l}
X_{i}(t+1)=X_{i}(t)+\frac{X_{j}(t)-X_{i}(t)}{\left\|X_{j}(t)-X_{i}(t)\right\|} \times \operatorname{Step} \times \operatorname{rand}() \frac{Y_{j}}{n_{f}}>\delta Y_{i}, \\
\text { equation (4), otherwise. }
\end{array}\right.
$$


(4) Random Behavior. The fish swim randomly in water; in fact, they are seeking food or companions in larger ranges. It is the default behavior of preying. The AF location is

$$
X_{i}(t+1)=X_{i}(t)+\text { Visual } \times(2 \times \operatorname{rand}()-1),
$$

where the terms are the same as above.

2.2.2. Improved ASFA. AFSA's system is based on the collective behaviors of AFs. An arguable weakness of the AF is its seeking ability in large or flat areas. More precisely, as the local optimum is searched and updated to its self-organized system [37] by an individual AF, others companions behave chaotically, thus reducing the seeking efficiency of the global optima.

To improve the performance of AFSA, a crossover operator is embedded into the AFSA, using genetic algorithm for reference. The crossover operator combines the features of two individuals and creates a potential better offspring. The search of the global optima is improved as well by allowing some uncertainty.

In every iteration, a specified number of $\mathrm{AF}$ are put in the pool, according to the crossover probability. Initially, the crossover is operated between every AF, in order to generate a corresponding child AF. The child AF replaces the parent $\mathrm{AF}$ and its position is given by the arithmetic crossover of the parent AF:

$$
X^{\text {child }}(t)=\operatorname{rand}() \times X_{1}^{\text {parent }}(t)+(1-\operatorname{rand}()) \times X_{2}^{\text {parent }}(t),
$$

where the terms are the same as above.

Finally, a new fish swarm is produced from the iterative procedure.

2.3. Artificial Neural Network (ANN). The artificial neural network (ANN) is a mathematical model vaguely inspired by the biological neural networks of animal brains [41]. The single hidden layer, feedforward neural network is the simplest unidirectional (input to output) form, consisting of three layers: input, hidden, and output. Figure 2 depicts this. Perhaps, the greatest advantage of this method (multi-layer perceptron with just one hidden layer), according to the universal approximation theorem, is its ability to approximate every (real-valued) bounded function [42]. It is therefore used for a wide range of activation functions, e.g., sigmoid function [43], and for both classification and regression problems. For regression, typically $l=1$, with one output unit $(\delta)$ at the top [44].

For dam deformation prediction, a popular approach is to first transform the raw observation data on dam deformation via the statistical model (equation (1)). Then, the features (hydrostatic components, temperature components, and time effect components) become the inputs of the neural network. Finally, the output becomes the observation data, $\delta$, from the deformation behavior.

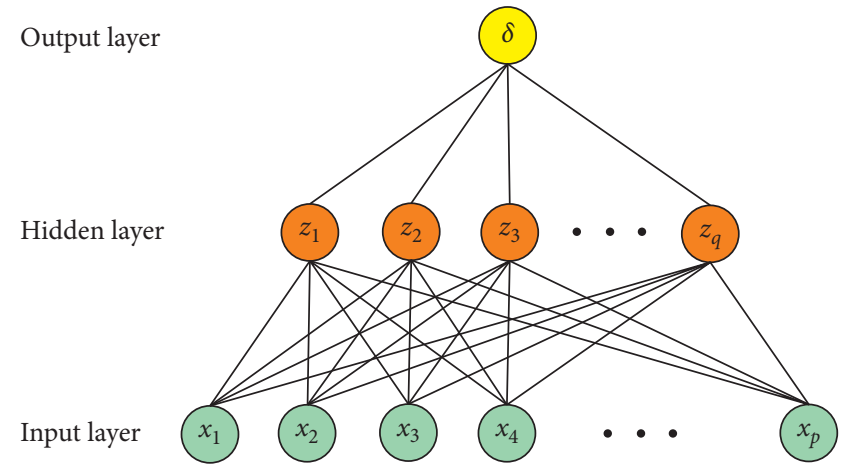

Figure 2: Schematic of a single hidden layer, feedforward neural network.

2.4. Backpropagation (BP) Algorithm. Backpropagation (BP) algorithm is a widely used algorithm in training feedforward neural networks. The backpropagation algorithm works by computing the gradient of the loss function with respect to each weight by the chain rule. More explicitly, it computes the gradient of one layer at a time by iterating backwards (from the last layer) to avoid redundant calculations from intermediate terms in the chain rule [45].

As seen in Figure 2, given the training set $\left\{\left(x_{1}^{\mathrm{HST}}, \delta_{1}\right)\right.$, $\left.\left(x_{2}^{\mathrm{HST}}, \delta_{2}\right), \ldots,\left(x_{N}^{\mathrm{HST}}, \delta_{N}\right)\right\}, x_{i}^{\mathrm{HST}} \in R^{p}, \delta_{i} \in R(\delta$ is the observation data on dam deformation) in the neural network, let there be $p$ input neurons, one output neuron, and $q$ hidden neurons.

The threshold value of the $j$ th hidden layer neuron is $\theta_{j}$. In a similar way, let $\alpha_{j}=\sum_{i=1}^{p} \omega_{i j} x_{i}$ be the input of the $j$ th hidden layer neuron, where $\omega_{i j}$ represents the connection weight from input neuron $i$ to hidden neuron $j$, and $\beta=\sum_{j=1}^{q} v_{j} b_{j}$, the input of the output layer neuron, where $v_{j}$ represents the connection weight from the hidden neuron $j$ to the output neuron and $b_{j}$ denotes the output of the $j$ th hidden layer neuron. Then, the network output of the $k$ th training data $\left(x_{k}^{\mathrm{HST}}, \delta_{k}\right)$ may be described as

$$
\widehat{\delta}_{k}=f\left(\beta_{k}-\gamma_{k}\right) \text {, }
$$

where $f$ is the activation function and $\gamma$ denotes the threshold value of the output layer neuron.

And the fitting error of the $k$ th training data is calculated:

$$
E_{k}=\frac{1}{2}\left(\widehat{\delta}_{k}-\delta_{k}\right)^{2} .
$$

The iterative procedure of the BP algorithm is described in the following steps:

(1) Compute the network output $\widehat{\delta}_{k}$.

(2) Calculate the gradient term of output layer neurons $g=-\partial E_{k} / \partial \widehat{\delta}_{k} \cdot \partial \widehat{\delta}_{k} / \partial \beta$.

(3) Calculate the gradient term of hidden layer neurons $g_{j}=-\partial E_{k} / \partial b_{j} \cdot \partial b_{j} / \partial \alpha_{j}$.

(4) Update the connection weights $\omega_{i j}, v_{j}$ and thresholds $\theta_{j}, \gamma$ according to the gradient descent strategy. Obtain the cumulative error of the training set fitted by the network as 


$$
E=\frac{1}{N} \sum_{k=1}^{N} E_{k}
$$

(5) Evaluate the stopping criteria. If satisfied, store the output; otherwise, repeat Steps 1-4.

\subsection{Hybrid IAFSA-BP-ANN Model for Predicting Dam De-} formation Behavior. The two AFSA and BP algorithms are combined into a hybrid-learning algorithm, referred to as IAFSA-BP, to overcome their standalone shortcomings. The IAFSA-BP algorithm's searching process starts with a random AF group, where the IAFSA algorithm is executed to attain the global best position. Subsequently, the BP algorithm is used to search for the global optimum, leading to an overall faster convergence and high accuracy results. The procedure of the IAFSA-BP neural network prediction model is summarized in Figure 3. The proposed model couples three stages.

In Stage 1, the statistical relations are studied via the statistical model. The input vectors of the neural network are obtained statistically; namely, input $=\left(H, H^{2}, \ldots\right.$, $\left.H^{n}, \sin x, \cos x, \sin 2 x, \cos 2 x, \theta, \ln \theta\right)$, where $x=2 \pi t / 365$.

In Stage 2, the ANN is trained on the training set and its performance is compared with the validation set.

Step 1. Determine the topology of the neural network. Initialize the weights and thresholds of the neural network in the range of $[0,0.1]$. Notice every AF represents a set of weights and thresholds of the neural network. The number of parameters to be identified is $p \times q+2 q+1$, as shown in Figure 2 .

Step 2. Implement the following behavior, swarming behavior and preying behavior (the default behavior).

Step 3. Use the function that calculates the validation error of basic BP neural network as the fitness function (food consistence) of IAFSA algorithm. IAFSA algorithm is used to train the weights and thresholds.

Step 4. Evaluate the behavior of AFs. Every artificial fish (AF) tries to find better food consistence position by simulating swarming behavior and following behavior, respectively. If this is successful, choose the better one to perform; otherwise, perform preying behavior.

Step 5. Operate the arithmetic crossover; then, generate the new fish swarm.

Step 6. If the maximal iteration times are arrived, go to Step 7; else, go to Step 2.

Step 7. Output the optimal weights and thresholds of the ANN.

After defining the training, validation, and testing set, with a number of examples, the ANN with the best validation set performance is chosen and demonstrated against the testing set. The solution of the maximum problem is taken as an example, and the reciprocal of validation set error is set as the food consistence of the fish swarm.

Ultimately, Stage 3 deals with the deformation simulation of the dam. $\delta(t+1), \delta(t+2), \ldots, \delta(t+?)$ is the prediction over the testing set. The schematics of the stages are presented in Figure 3.

\section{Case Study}

The data used for the study correspond to a concrete arch dam, located on the Yalong River in China. It is a double curvature arch dam, completed in 2014, made up of 26 sections, with a maximum dam height of $305 \mathrm{~m}$. The width of the dam's crest and bottom is $16 \mathrm{~m}$ and $63 \mathrm{~m}$, respectively. The reservoir capacity is 7.76 billion $\mathrm{m}^{3}$.

Figure 4 depicts the dam, as well as the location of the monitoring device. Using the available records, the study focused on the radial displacements measured by pendulum PL13-1 (along the upstream-downstream direction). Figure 5 shows the records, divided into three sets: training (first $80 \%$ sample), validation (middle $10 \%$ sample), and testing (last $10 \%$ sample); the displacements toward upstream are negative, and towards downstream, positive.

The training set is used to calculate gradients and update connection weights and threshold values of ANN. In the validation range, the output error is the food consistence (fitness function) in the current position of each AF; namely, food_consistence $=1 /$ error $_{\text {validation}}$, where error $_{\text {validation }}$ is typically the sum of the squared errors. In the model, the objective of pursuing the error's minimum is converted to the maximum value of food consistence.

The goodness of the model accuracy is computed in terms of the root mean squared error (RMSE), a measure of the differences between predicted values and observed values. The magnitude and the deviation of the target variable are determined with the average relative variance (ARV) $[18,46]$. They are defined as

$$
\begin{aligned}
& \delta_{\mathrm{RMSE}}=\sqrt{\frac{1}{n} \sum_{i=1}^{n}\left(\delta_{i}-\widehat{\delta}_{i}\right)^{2},} \\
& \delta_{\mathrm{ARV}}=\frac{\sum_{i=1}^{n}\left(\delta_{i}-\widehat{\delta}_{i}\right)^{2}}{\sum_{i=1}^{n}\left(\delta_{i}-\widehat{\delta}_{i}\right)^{2}}=\frac{\delta_{\mathrm{MSE}}}{\sigma^{2}},
\end{aligned}
$$

where $n$ is the length of the analyzed data set; $\delta_{i}$ is the observation on deformation behavior; $\widehat{\delta}_{i}$ is the predicted values; $\bar{\delta}$ is the observation mean; $\delta_{\mathrm{MSE}}$ is the mean square error, and $\delta_{\mathrm{MSE}}=(1 / n) \sum_{i=1}^{n}\left(\delta_{i}-\widehat{\delta}_{i}\right)^{2}$; and $\sigma^{2}$ is the variance of the analyzed data set.

The statistical model is the most widely applied in dam engineering [5]. Through the statistical model, the wide knowledge in analyzing the behavior of concrete dams provides the prior knowledge between the loads and the deformation. It will be helpful for the data-based models' prediction accuracy, such as ANN. Thus, according to the statistical relations between the loads and the deformation (equation (1)), input $=\left(H, H^{2}, H^{3}, \quad H^{4}, \sin 2 \pi t / 365\right.$, $\cos 2 \pi t / 365, \sin 4 \pi t / 365, \cos 4 \pi t / 365, \theta, \ln \theta)$ is regarded as ANN inputs.

Hyper-parameters (for example, number of hidden layers and units, activation function) play an important role 


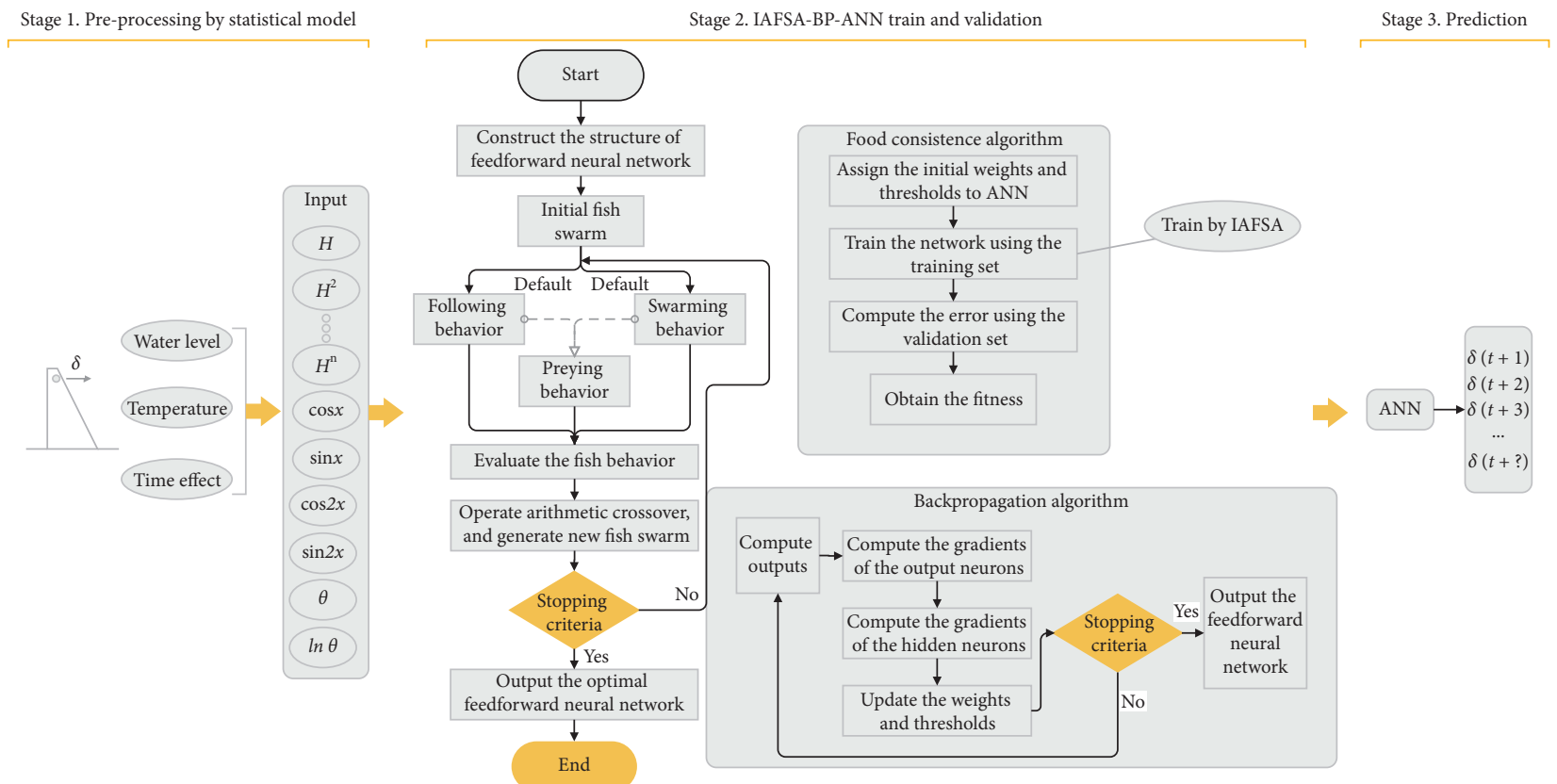

FIGURE 3: Schematic diagram of the proposed model.

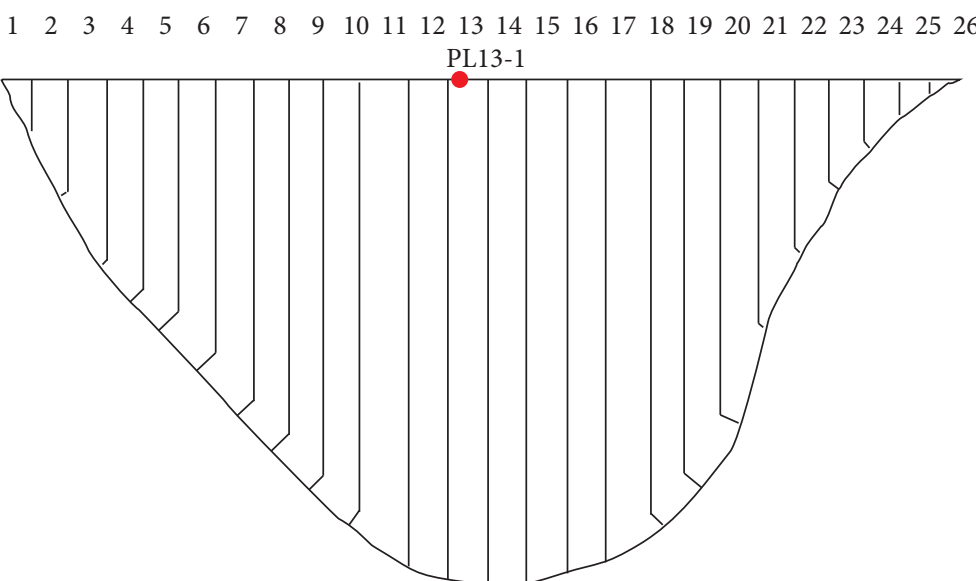

(a)

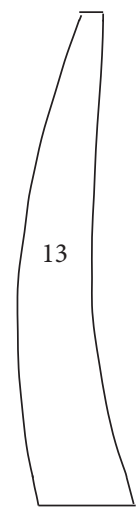

(b)

Figure 4: Geometry and location of the monitoring device. (a) Left: view from downstream; (b) highest cross section. To be continued.

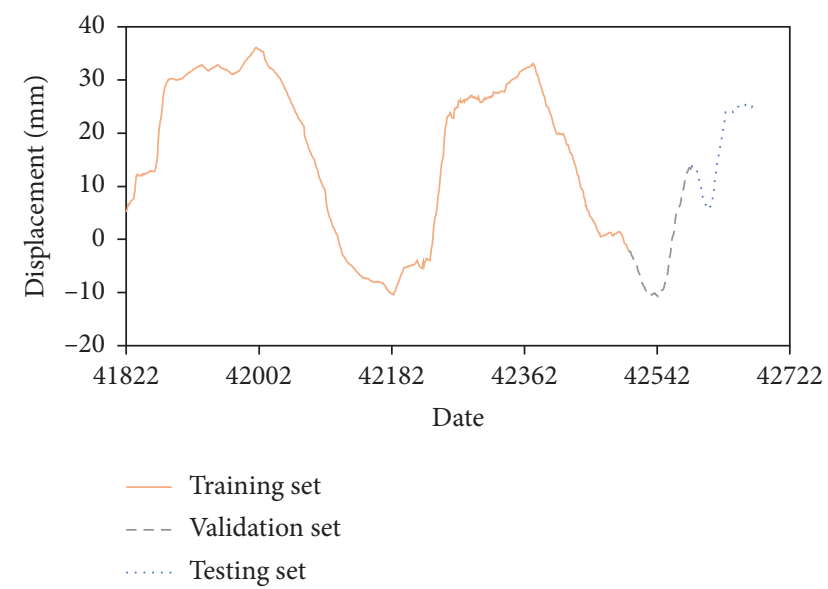

FIGURE 5: Displacement recorded by the pendulum at block 13. in the performance of the ANN $[47,48]$. Because the robustness of the neural network is influenced by the hidden layer, in order to achieve better accuracy, the Hecht-Nielson method [49] is adopted to determine the neuron number of the hidden layer; thus, when the neuron number of the input layer is $p$, the neuron number of the hidden layer is $q=2 p+1$. The ANN of the study comprised an input layer with 10 parameters (input), a hidden layer with 21 nodes, and an output layer with one parameter (to denote the upstream-downstream radial displacement of the PL13-1 pendulum). Thus, the neural network structure is 10-21-1.

The hidden layer's activation function was assumed as a sigmoid function (equation (15)), and the output layer's activation function was a linear function (equation (16)). The Levenberg-Marquardt algorithm was used in the training process: 


$$
\begin{aligned}
& y=\frac{1}{1+e^{-x}}, \\
& y=x .
\end{aligned}
$$

Considering that the AF's position represents the connection weights and thresholds of the ANN, every AF consisted of $p \times q+2 q+1=253$ parameters. The component varies from 0 to 1 . Table 1 provides the values for other IAFSA parameters.

\section{Results and Discussion}

4.1. Prediction Results. Figure 6 presents the optimization process of the IAFSA, which is also the minimal validation set errors varying with the iteration times. When the iteration time reached 6 , the validation error converged. IAFSA-BP-ANN predicted results versus observations for PL13-1 is shown in Figure 7. Figure 7 compares observed and computed displacements of training set, validation set, and testing set obtained by IAFSA-BP-ANN for PL13-1 pendulum. It provides an intuition on the good performance on the training set, validation set, and testing set.

4.2. Model Comparison. The prediction accuracy and potential of the proposed model are verified against four popular methods: the statistical model, the backpropagation neural network optimized by GA (GA-BP-ANN), the backpropagation neural network optimized by PSO (PSOBP-ANN), and the backpropagation neural network optimized by AFSA (AFSA-BP-ANN).

Due to the lack of validation in the statistical model, its training is set to $90 \%$ of the sample, compared with the remaining models' $80 \%$. It is crucial to bear in mind the conceivable bias in the comparisons, since increasing the amount of training leads to a better performance. The structure 10-21-1 was adopted for the GA-BP-ANN, PSOBP-ANN, AFSA-BP-ANN, and IAFSA-BP-ANN models. Ceteris paribus, the higher the accuracy, the better the model.

Figure 8 depicts the optimization processes of the models, except for the statistical model. The IAFSA has a convergence after 6 iterations (Error $=0.6$ ), the fastest of all, with a trend being similar to but slightly less accurate (higher error) than the AFSA. The converged error of the PSO is relatively high, about 2.5, although its convergence speed is modest (19 iterations). The GA performs the worst, by attaining a convergence after 45 iterations, with a mean squared error $\left(\delta_{\mathrm{MSE}}\right)$ of 17.

For reasons of comparison clarity, only the predicted outputs of the testing set and their residuals are displayed in Figures 9 and 10, respectively. IAFSA-BP-ANN's predictions can be seen from the testing period of Figure 7. All the models capture the overall trend of the testing set, although the accuracy varies to a large extent, according to the model and the range of the testing set. For example, the PSO predicts poorly the curve at first-half stage but then improves
TABLE 1: Parameters of IAFSA.

\begin{tabular}{lc}
\hline Parameter & Value \\
\hline Number of AF & 50 \\
Visual distance of AF & 3 \\
Crowd factor & 0.618 \\
Step of AF & 0.5 \\
Trying number of preying behavior & 20 \\
Number of iterations & 20 \\
Crossover probability & 0.9
\end{tabular}

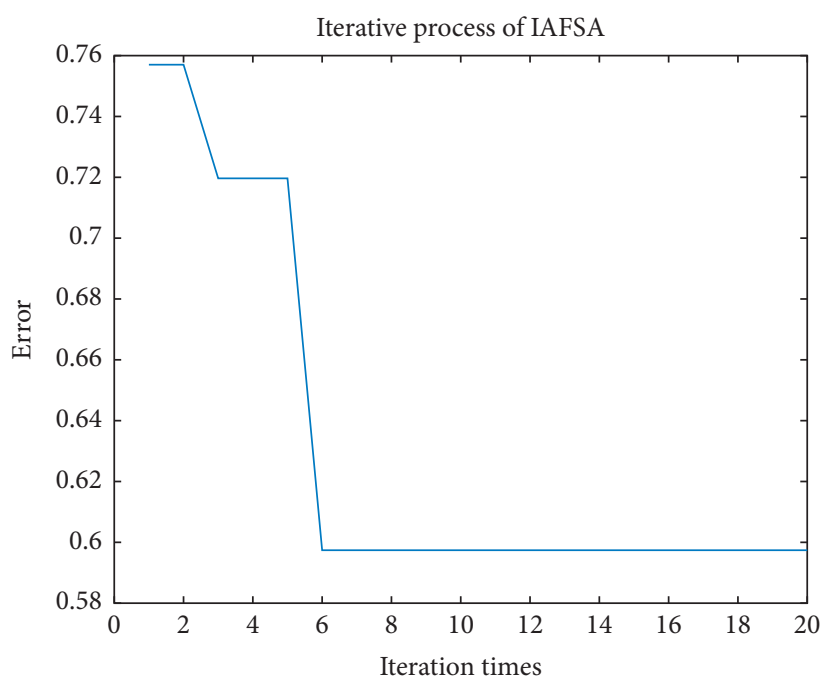

FIgURE 6: Optimization process of IAFSA.

dramatically, having minimal, if any at some points, residuals (Figure 10(c)). On the contrary, the modest predictions of the statistical model deteriorate slightly over time (Figure 10(a)). The GA predicts accurately the last portion of the observations but underpredicts beforehand. The AFSA captures the beginning and end relatively well but fails to capture the intermediate process and initiation of the plateau. The IAFSA yields good results and contains the least error deviations (low overall residuals, see Figure 10(e)).

To analyze the results in a quantitative way, two performance indices (equations (13) and (14)) are adopted in this study. In Table 2, a comparison of the set fitting is made between the four methods, in terms of their root mean squared error $\left(\delta_{\mathrm{RMSE}}\right)$ and average relative variance $\left(\delta_{\mathrm{ARV}}\right)$. The lowest values are in bold fonts.

It is apparent from this table that a good fitting to the training set does not consequently result in accurate predictions of validation, or a good validation into reliable simulations of testing. For example, the GA-BP-ANN showed the best performance for the training set but was the worst for validation (overfitting). The validation errors of the AFSA-BP-ANN were the smallest but similar to the PSOBP-ANN's, AFSA-BP-ANN's, and IAFSA-BP-ANN's. The IAFSA-BP-ANN, the proposed model, achieved the best testing results, even if its training yielded the worst but moderate results. 


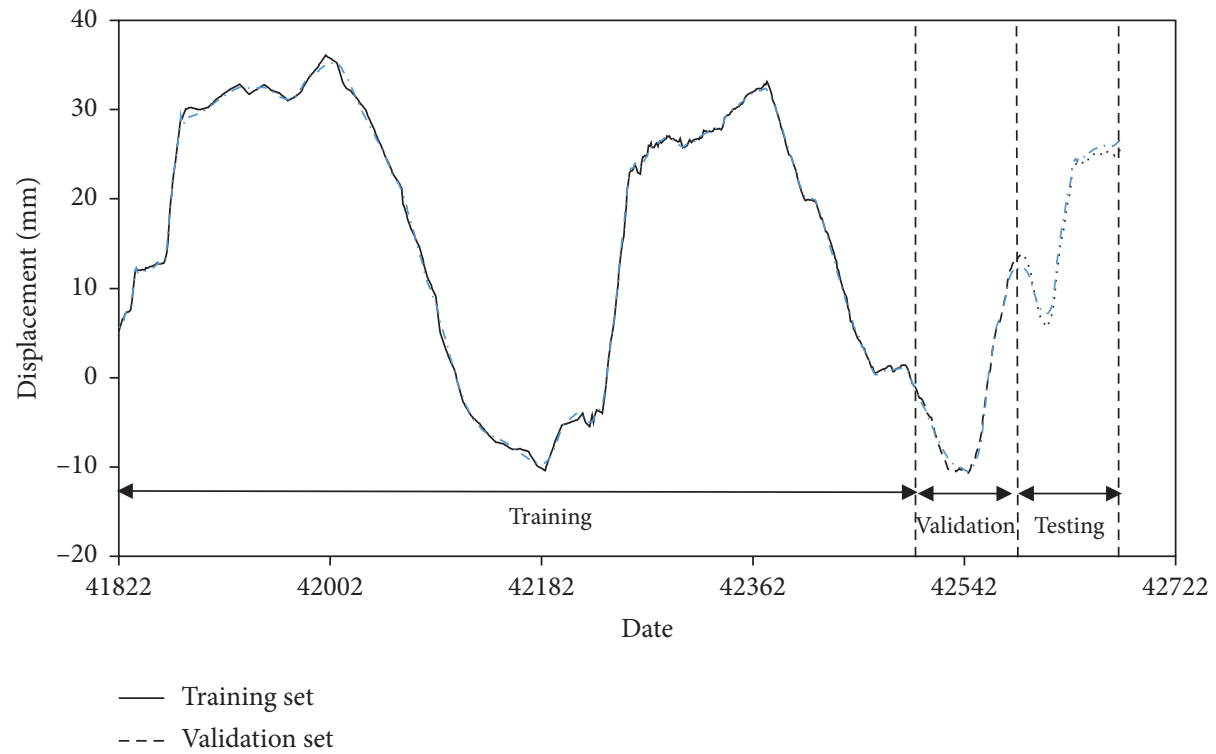

FIGURE 7: IAFSA-BP-ANN outputs (blue line) versus observations (black lines) for PL13-1.

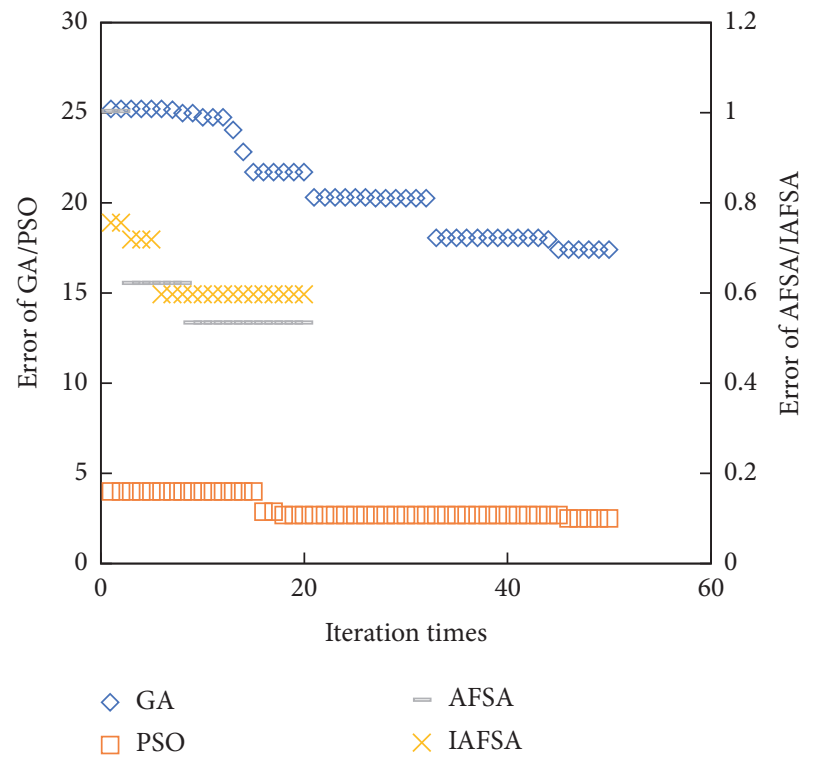

Figure 8: Optimization process of GA-BP-ANN, PSO-BP-ANN, AFSA-BP-ANN, and IAFSA-BP-ANN.
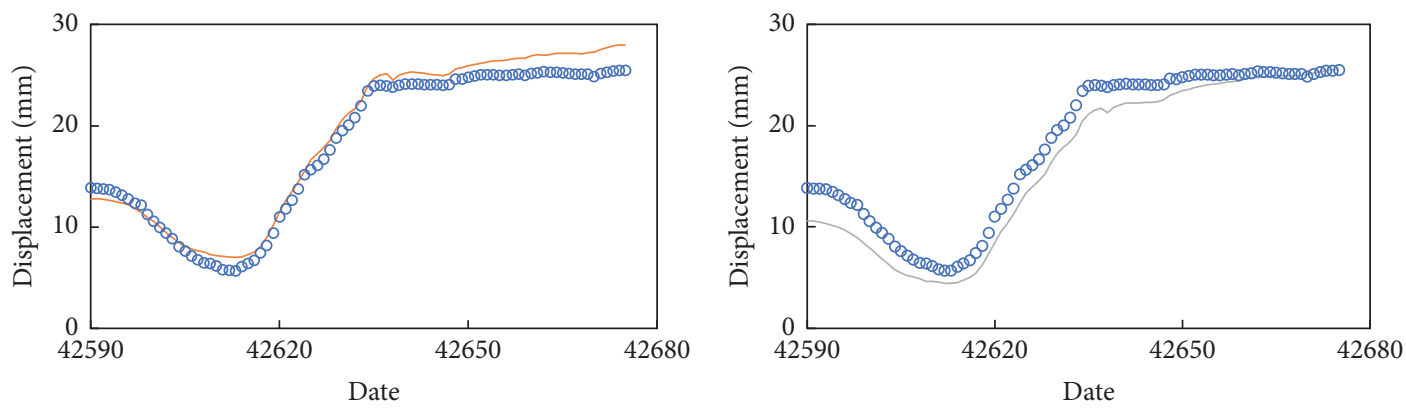

- Observation

- Observation

— Statistical model

- GA

(a)

Figure 9: Continued. 


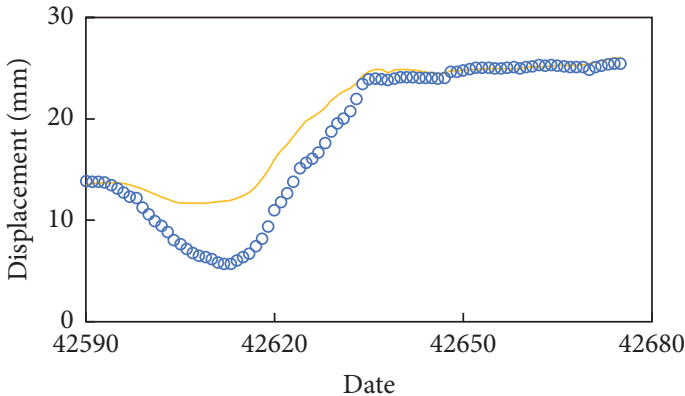

- Observation

- PSO

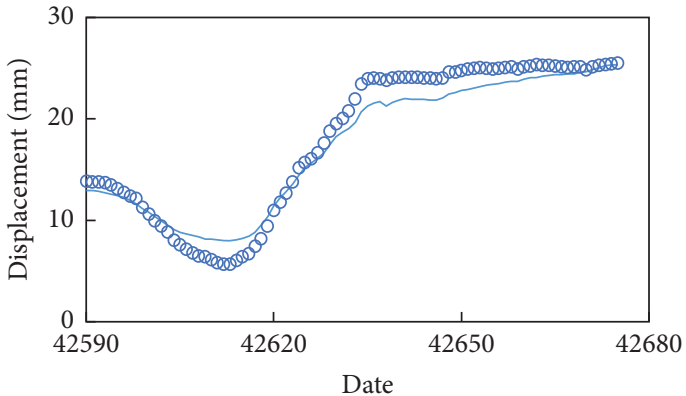

- Observation

— AFSA

(c)

(d)

Figure 9: Predicted displacements on testing set: (a) statistical model; (b) GA-BP-ANN; (c) PSO-BP-ANN; (d) AFSA-BP-ANN.

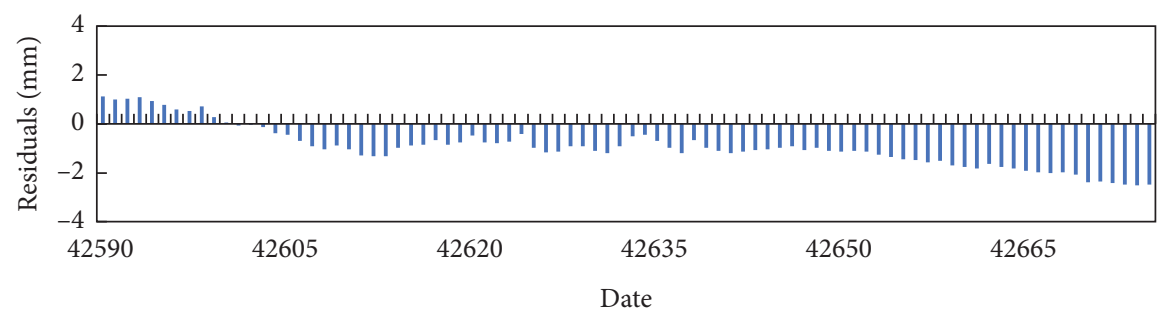

(a)

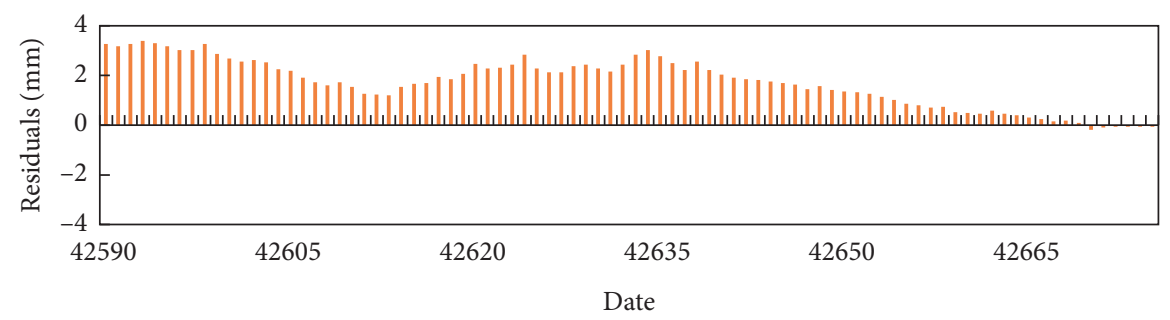

(b)

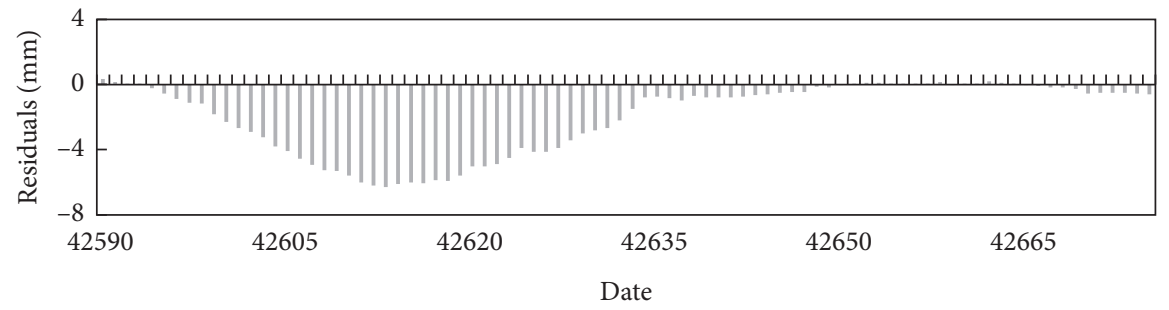

(c)

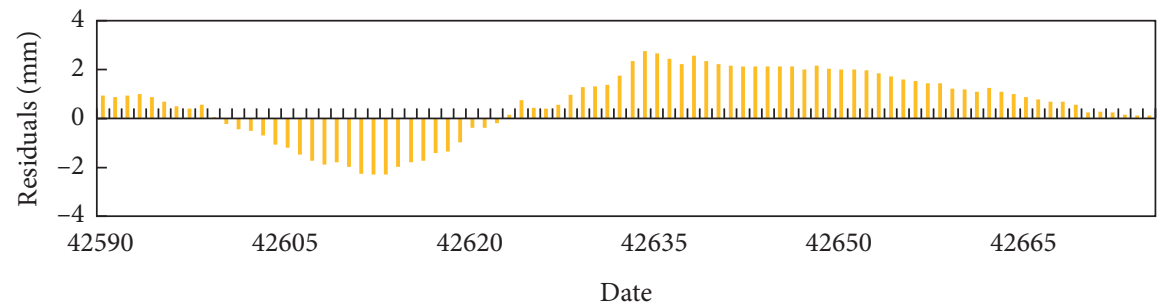

(d)

Figure 10: Continued. 


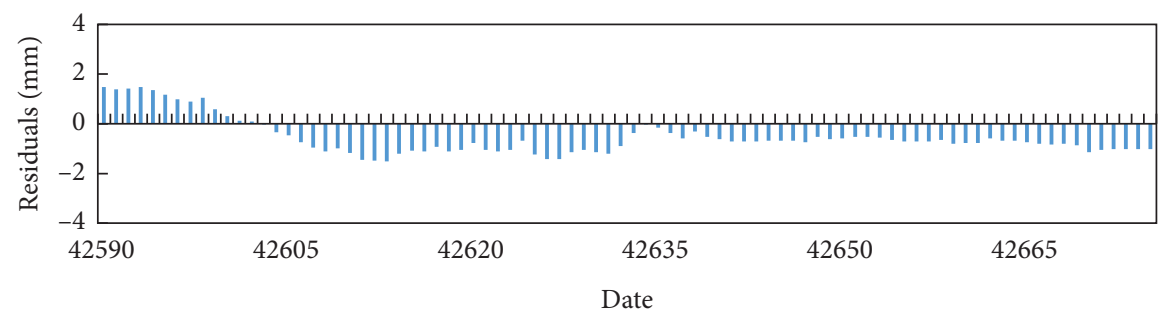

(e)

FIGURE 10: Residuals between model predictions and observations: (a) statistical model; (b) GA-BP-ANN; (c) PSO-BP-ANN; (d) AFSA-BPANN; (e) IAFSA-BP-ANN.

TAble 2: Performance indices for statistical model, GA-BP-ANN, PSO-BP-ANN, AFSA-BP-ANN, and IAFSA-BP-ANN.

\begin{tabular}{lcccrrr}
\hline & \multicolumn{2}{c}{ Training set } & \multicolumn{2}{c}{ Validation set } & \multicolumn{2}{c}{ Testing set } \\
& $\delta_{\text {RMSE }}$ & $\delta_{\text {ARV }}$ & $\delta_{\text {RMSE }}$ & $\delta_{\text {ARV }}$ & $\delta_{\text {RMSE }}$ & 1.2712 \\
\hline Statistical model & & & & & 0.0297 \\
GA-BP-ANN & $\mathbf{0 . 3 2 9 0}$ & $\mathbf{0 . 0 0 0 5}$ & 17.5116 & 4.5665 & 1.9816 \\
PSO-BP-ANN & 0.3836 & 0.0007 & 0.5720 & 0.0049 & 2.9266 \\
AFSA-BP-ANN & 0.3595 & 0.0006 & $\mathbf{0 . 5 3 4 3}$ & $\mathbf{0 . 0 0 4 3}$ & 1.4953 \\
IAFSA-BP-ANN & 0.4217 & 0.0008 & 0.5974 & 0.0053 & 0.1576 \\
\hline
\end{tabular}

TABle 3: Performance indices of residuals for all the models.

\begin{tabular}{lcc}
\hline Model & $\sigma_{\varepsilon}$ & $\sum_{i=1}^{n} \varepsilon_{i}^{2}$ \\
\hline Statistical model & 0.8559 & 138.98 \\
GA-BP-ANN & 0.9894 & 337.69 \\
PSO-BP-ANN & 2.1942 & 736.59 \\
AFSA-BP-ANN & 1.3788 & 192.28 \\
IAFSA-BP-ANN & $\mathbf{0 . 7 3 0 2}$ & $\mathbf{7 2 . 1 1}$ \\
\hline
\end{tabular}

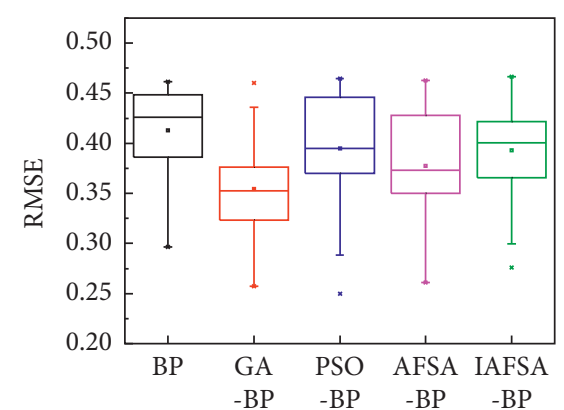

(a)

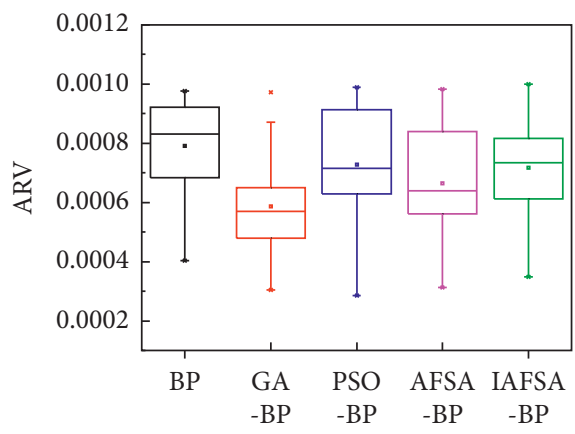

(d)

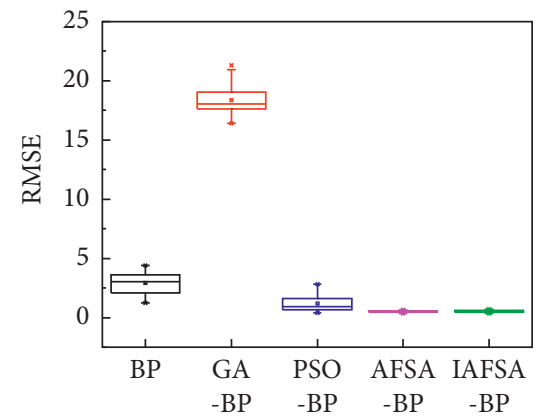

(b)

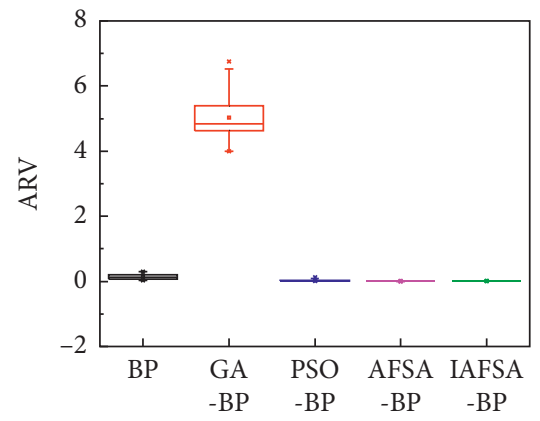

(e)

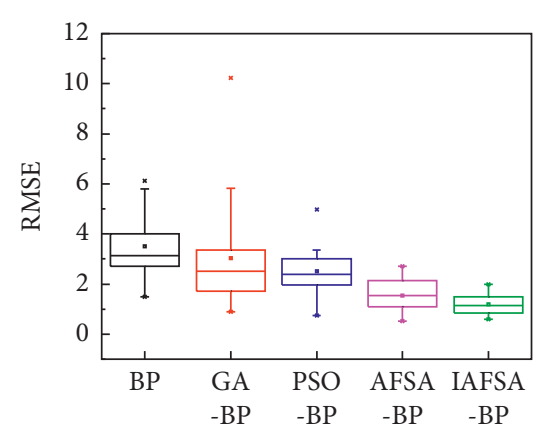

(c)

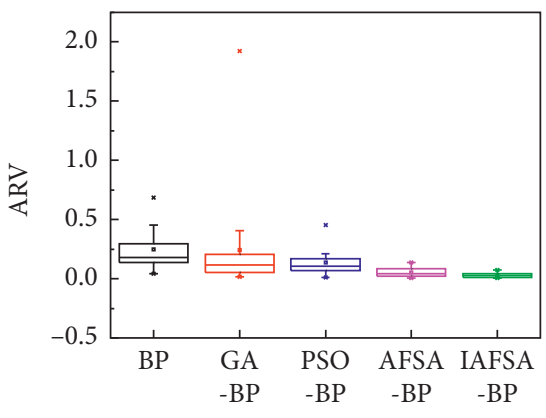

(f)

FIGURE 11: Solution distribution of 30 independent runs by using BP-ANN, GA-BP-ANN, PSO-BP-ANN, AFSA-BP-ANN, and IAFSA-BPANN, respectively: (a) $\delta_{\text {RMSE }}$ over training set; (b) $\delta_{\text {RMSE }}$ over validation set; (c) $\delta_{\text {RMSE }}$ over testing set; (d) $\delta_{\text {ARV }}$ over training set; (e) $\delta_{\text {ARV }}$ over validation set; (f) $\delta_{\text {ARV }}$ over testing set. 
TABLE 4: Statistical comparison of $\delta_{\text {RMSE }}$ for BP-ANN, GA-BP-ANN, PSO-BP-ANN, AFSA-BP-ANN, and IAFSA-BP-ANN based on 30 independent runs.

\begin{tabular}{lcccccc}
\hline & \multicolumn{2}{c}{ Training set } & \multicolumn{2}{c}{ Validation set } & \multicolumn{2}{c}{ Testing set } \\
& Mean & $p$ value & Mean & $p$ value & Mean & \multicolumn{2}{c}{$p$ value } \\
\hline BP-ANN & 0.4131 & 0.0854 & 2.9166 & $4.03 E-21$ & 3.5042 & $3.0255-15$ \\
GA-BP-ANN & $\mathbf{0 . 3 5 4 2}$ & 0.0035 & 18.3578 & $7.16 E-63$ & $2.89 E-06$ \\
PSO-BP-ANN & 0.3950 & 0.8692 & 1.2097 & $4.58 E-06$ & 2.5101 & $2.64 E-08$ \\
AFSA-BP-ANN & 0.3776 & 0.2358 & 0.5290 & 0.7919 & 1.5415 & 0.0169 \\
IAFSA-BP-ANN & 0.3929 & - & $\mathbf{0 . 5 2 3 5}$ & - & $\mathbf{1 . 1 9 0 7}$ \\
\hline
\end{tabular}

TABLE 5: Statistical comparison of $\delta_{\mathrm{ARV}}$ for BP-ANN, GA-BPANN, PSO-BP-ANN, AFSA-BP-ANN, and IAFSA-BP-ANN based on 30 independent runs.

\begin{tabular}{lcccccc}
\hline & \multicolumn{2}{c}{ Training set } & \multicolumn{2}{c}{ Validation set } & \multicolumn{2}{c}{ Testing set } \\
& Mean & $p$ & Mean & $p$ value & Mean & $p$ value \\
& value & & & & \\
& & & & & & \\
BP- & 0.0008 & 0.0805 & 0.1381 & $3.15 E-13$ & 0.2496 & $2.91 E-09$ \\
ANN & & & & & & \\
GA- & & & & & & \\
BP- & $\mathbf{0 . 0 0 0 6}$ & 0.0037 & 5.0369 & $6.34 E-46$ & 0.2409 & 0.0032 \\
ANN & & & & & & \\
$\begin{array}{l}\text { PSO- } \\
\text { BP- }\end{array}$ & 0.0007 & 0.8205 & 0.0296 & 0.0002 & 0.1353 & $3.81 E-06$ \\
ANN & & & & & & \\
$\begin{array}{l}\text { AFSA- } \\
\text { BP- }\end{array}$ & 0.0007 & 0.2411 & $\mathbf{0 . 0 0 4 2}$ & 0.8318 & 0.0514 & 0.0083 \\
ANN & & & & & & \\
$\begin{array}{l}\text { IAFSA- } \\
\text { BP- }\end{array}$ & 0.0007 & - & $\mathbf{0 . 0 0 4 2}$ & - & $\mathbf{0 . 0 2 9 2}$ & - \\
ANN & & & & & & \\
\hline
\end{tabular}

4.3. Discussion. The variance $\left(\sigma_{\varepsilon}\right)$ of the residuals $\left(\varepsilon_{i}\right)$ for the five models is given in Table 3 . As observed, the two statistical estimators, $\sigma_{\varepsilon}$ and $\sum_{i=1}^{n} \varepsilon_{i}{ }^{2}$, of the IAFSA-BP-ANN are the lowest, thus implying the narrowest confidence interval under the same level of significance. In light of the good prediction accuracy, the IAFSA-BP-ANN can be adopted for a health evaluation of the dam's deformation behavior, in order to obtain good performance and less false alarms.

It is sometimes commented that the proposed model is not based on the principles of mechanics but only the knowledge of statistical nature. Although this may be true, nevertheless, the physical behavior is intrinsic to the observed values (i.e., the observations are statistical data but are still governed by the actual behavior of the structure [22]), and thus, the statistical-based model predictions are reasonable and practical.

To more precisely assess the performance of each optimization algorithm, they are tested for 30 independent runs and their performance indices $\left(\delta_{\mathrm{RMSE}}\right.$ and $\left.\delta_{\mathrm{ARV}}\right)$ are calculated. BP-ANN is taken to highlight the effect of IAFSA. Then, solution distribution of 30 independent runs by using BP-ANN, GA-BP-ANN, PSO-BP-ANN, AFSA-BP-ANN, and IAFSA-BP-ANN is depicted in Figure 11.

Tables 4 and 5 show the mean values of the performance indices and the $p$ values of IAFSA in comparison with other algorithms. The best values of performance indices are highlighted in boldface in Tables 4 and 5. There is not a great difference between performance indices for all the algorithms on the training set. The validation results are similar except GA-BP, which is overfitting. From them, we can see that testing accuracy of IAFSA-BP-ANN outperforms other algorithms.

One-way analysis of variance is adopted to detect the differences between the behaviors of IAFSA and the compared algorithm (Tables 4 and 5). A $p$ value smaller than 0.05 signifies significant difference between IAFSA and the compared algorithm. According to Figure 11 and Tables 4 and 5, IAFSA shows better generalization ability than the other algorithms. The results are consistent with the former analysis.

\section{Conclusions}

In this paper, an IAFSA-BP-ANN model was proposed to forecast the dam deformation behavior, specifically the crest radial displacements. The input of the model was generated from the statistical relations between loads and deformation behavior of dams. The IAFSA was chosen for its global search ability, and the BP, for its local search ability. The combined algorithm overcame their standalone shortcomings. A case dam study was used to verify the model, along with other four methods: statistical model, GA-BP-ANN, PSO-BP-ANN, and AFSA-BP-ANN. The superiority of the proposed model was demonstrated in a quantitative manner (error graph, root mean squared error $\delta_{\mathrm{RMSE}}$, average relative variance $\delta_{\mathrm{ARV}}$, etc.).

The main advantage of the IAFSA-BP-ANN was its high prediction accuracy, fast convergence speed, and low residuals performance indices, thus, implying a narrow confidence interval and less dam's false warnings. Additionally, the proposed model is linked with engineering experience, namely, the most popular data-driven model, the statistical model.

As for dam safety monitoring, the data-based behavior models should never be the only source of information to make assessment. Law-based behavior models, such as finite element analysis, should be taken into account. In future works, the model effectiveness can be tested with more available dam information to provide greater insights into dam deformation behavior.

\section{Data Availability}

The monitoring data of the ultrahigh arc dam used to support the findings of this study are available from the corresponding author upon request. 


\section{Conflicts of Interest}

The authors declare that there are no conflicts of interest regarding the publication of this paper.

\section{Acknowledgments}

This work was funded by the National Natural Science Foundation of China (Grant nos. 51739003 and 51909173), Free Exploration Project of Hohai University (no. B200201058), and Open Foundation of Changjiang Survey, Planning, Design and Research Co., Ltd. (CX2019K01).

\section{References}

[1] M. L. eKay and G. H. McClelland, "Predicting loss of life in cases of dam failure and flash flood," Risk Analysis, vol. 13, no. 2, pp. 193-205, 1993.

[2] C. Gu and Z. Wu, Safety Monitoring of Dams and Dam Foundations-Theories \& Methods and Their Application, Hohai University Press, Nanjing, China, 2006, (in Chinese).

[3] H. Su, X. Li, B. Yang, and Z. Wen, "Wavelet support vector machine-based prediction model of dam deformation," $\mathrm{Me}$ chanical Systems and Signal Processing, vol. 110, pp. 412-427, 2018.

[4] F. Salazar, M. A. Toledo, E. Oñate, and R. Morán, “An empirical comparison of machine learning techniques for dam behaviour modelling," Structural Safety, vol. 56, pp. 9-17, 2015.

[5] J. Mata, A. Tavares de Castro, and J. Sá da Costa, "Constructing statistical models for arch dam deformation," Structural Control and Health Monitoring, vol. 21, no. 3, pp. 423-437, 2014.

[6] M. Li, Y. Shen, Q. Ren, and H. Li, "A new distributed time series evolution prediction model for dam deformation based on constituent elements," Advanced Engineering Informatics, vol. 39, pp. 41-52, 2019.

[7] H. Salehi and R. Burgueño, "Emerging artificial intelligence methods in structural engineering," Engineering Structures, vol. 171, pp. 170-189, 2018.

[8] B. Stojanovic, M. Milivojevic, M. Ivanovic, N. Milivojevic, and D. Divac, "Adaptive system for dam behavior modeling based on linear regression and genetic algorithms," Advances in Engineering Software, vol. 65, pp. 182-190, 2013.

[9] A. Majdi and M. Beiki, "Evolving neural network using a genetic algorithm for predicting the deformation modulus of rock masses," International Journal of Rock Mechanics and Mining Sciences, vol. 47, no. 2, pp. 246-253, 2010.

[10] G. Venter and J. Sobieszczanski-Sobieski, "Particle swarm optimization," AIAA Journal, vol. 41, no. 8, pp. 1583-1589, 2003.

[11] S. Gao, Y. Yu, Y. Wang, J. Wang, J. Cheng, and M. Zhou, "Chaotic local search-based differential evolution algorithms for optimization," IEEE Transactions on Systems, Man, and Cybernetics: Systems, 2020.

[12] Y. Wang, Y. Yu, S. Gao, H. Pan, and G. Yang, "A hierarchical gravitational search algorithm with an effective gravitational constant," Swarm and Evolutionary Computation, vol. 46, pp. 118-139, 2019.

[13] G. Zong Woo, K. Joong Hoon, and G. V. Loganathan, "A new heuristic optimization algorithm: harmony search," Simulation, vol. 76, no. 2, pp. 60-68, 2001.
[14] A. Lee, Z. Geem, and K.-D. Suh, "Determination of optimal initial weights of an artificial neural network by using the harmony search algorithm: application to breakwater armor stones," Applied Sciences, vol. 6, no. 6, p. 164, 2016.

[15] V. Ranković, N. Grujović, D. Divac, and N. Milivojević, "Development of support vector regression identification model for prediction of dam structural behaviour," Structural Safety, vol. 48, pp. 33-39, 2014.

[16] T. Zhou, S. Gao, J. Wang, C. Chu, Y. Todo, and Z. Tang, "Financial time series prediction using a dendritic neuron model," Knowledge-Based Systems, vol. 105, pp. 214-224, 2016.

[17] S. Gao, M. Zhou, Y. Wang, J. Cheng, H. Yachi, and J. Wang, "Dendritic neuron model with effective learning algorithms for classification, approximation, and prediction," IEEE Transactions on Neural Networks and Learning Systems, vol. 30, no. 2, pp. 601-614, 2019.

[18] F. Salazar, M. A. Toledo, E. Oñate, and B. Suárez, "Interpretation of dam deformation and leakage with boosted regression trees," Engineering Structures, vol. 119, pp. 230-251, 2016.

[19] F. Salazar, R. Morán, M. Á. Toledo, and E. Oñate, "Data-based models for the prediction of dam behaviour: a review and some methodological considerations," Archives of Computational Methods in Engineering, vol. 24, no. 1, pp. 1-21, 2017.

[20] B. Dai, C. Gu, E. Zhao, K. Zhu, W. Cao, and X. Qin, "Improved online sequential extreme learning machine for identifying crack behavior in concrete dam," Advances in Structural Engineering, vol. 22, no. 2, pp. 402-412, 2019.

[21] F. Kang, "Concrete dam deformation prediction model for health monitoring based on extreme learning machine," Structural Control and Health Monitoring, vol. 24, no. 10, p. e1997, 2017.

[22] J. Mata, "Interpretation of concrete dam behaviour with artificial neural network and multiple linear regression models," Engineering Structures, vol. 33, no. 3, pp. 903-910, 2011.

[23] Z. Yang, M. Mourshed, K. Liu, X. Xu, and S. Feng, "A novel competitive swarm optimized RBF neural network model for short-term solar power generation forecasting," Neurocomputing, vol. 397, 2020.

[24] M. Li, W. Si, Q. Ren, L. Song, and H. Liu, "An integrated method for evaluating and predicting long-term operation safety of concrete dams considering lag effect," Engineering with Computers, pp. 1-15, 2020.

[25] R. Fedele, G. Maier, and B. Miller, "Health assessment of concrete dams by overall inverse analyses and neural networks," International Journal of Fracture, vol. 137, no. 1-4, pp. 151-172, 2006.

[26] Y. Zhu, C. Gu, E. Zhao, J. Song, and Z. Guo, "Structural safety monitoring of high arch dam using improved ABC-BP model," Mathematical Problems in Engineering, vol. 2016, p. 9, Article ID 6858697, 2016.

[27] S. Chen, C. Gu, C. Lin, E. Zhao, and J. Song, "Safety monitoring model of a super-high concrete dam by using RBF neural network coupled with kernel principal component analysis," Mathematical Problems in Engineering, vol. 2018, Article ID 1712653, 13 pages, 2018.

[28] D. E. Rumelhart, G. E. Hinton, and R. J. Williams, "Learning representations by back-propagating errors," Nature, vol. 323, no. 6088, pp. 533-536, 1986.

[29] Y. Zhang, Y. Xu, Y. Zheng et al., "Multiobjective optimization design and experimental investigation on the axial flow pump with orthogonal test approach," Complexity, vol. 2019, Article ID 1467565, 14 pages, 2019. 
[30] J. Li, Brief Introduction of Back Propagation (BP) Neural Network Algorithm and its Improvement, Springer, Berlin, Germany, 2012.

[31] Y. LeCun, Y. Bengio, and G. Hinton, "Deep learning," Nature, vol. 521, no. 7553, pp. 436-444, 2015.

[32] M. Gori and A. Tesi, "On the problem of local minima in backpropagation," IEEE Transactions on Pattern Analysis and Machine Intelligence, vol. 14, no. 1, pp. 76-86, 1992.

[33] W. Bi, "Avoiding the local minima problem in backpropagation algorithm with modified error function," IEICE Transactions on Fundamentals of Electronics, Communications and Computer Sciences, vol. E88-A, no. 12, pp. 36453653, 2005.

[34] X. Wang, K. Yang, and C. Shen, "Study on MPGA-BP of gravity dam deformation prediction," Mathematical Problems in Engineering, vol. 2017, Article ID 2586107, 13 pages, 2017.

[35] X. Li, A New Intelligent Optimization-Artificial Fish Swarm Algorithm, Zhejiang University, Hangzhou, China, 2003.

[36] M. Neshat, G. Sepidnam, M. Sargolzaei, and A. N. Toosi, "Artificial fish swarm algorithm: a survey of the state-of-theart, hybridization, combinatorial and indicative applications," Artificial Intelligence Review, vol. 42, no. 4, pp. 965-997, 2014.

[37] W. Shen, X. Guo, C. Wu, and D. Wu, "Forecasting stock indices using radial basis function neural networks optimized by artificial fish swarm algorithm," Knowledge-Based Systems, vol. 24 , no. 3, pp. 378-385, 2011.

[38] H.-z. Su, Z.-r. Wu, and Z.-p. Wen, "Identification model for dam behavior based on wavelet network," Computer-Aided Civil and Infrastructure Engineering, vol. 22, no. 6, pp. 438448, 2007.

[39] N. Fang, J. Zhou, R. Zhang, Y. Liu, and Y. Zhang, "A hybrid of real coded genetic algorithm and artificial fish swarm algorithm for short-term optimal hydrothermal scheduling," International Journal of Electrical Power \& Energy Systems, vol. 62, pp. 617-629, 2014.

[40] C. W. Reynolds, "Flocks, herds and schools: a distributed behavioral model," in Proceedings of the 14th Annual Conference on Computer Graphics and Interactive Techniques, Anaheim, CA, USA, July 1987.

[41] Y.-Y. Chen, Y.-H. Lin, C.-C. Kung, M.-H. Chung, and I.-H. Yen, "Design and implementation of cloud analyticsassisted smart power meters considering advanced artificial intelligence as edge analytics in demand-side management for smart homes," Sensors, vol. 19, no. 9, p. 2047, 2019.

[42] B. C. Csáji, Approximation With Artificial Neural Networks, Faculty of Sciences, Etvs Lornd University, vol. 24, no. 48, p. 7, Hungary, Europe, 2001.

[43] G. Cybenko, "Approximation by superpositions of a sigmoidal function," Mathematics of Control, Signals, and Systems, vol. 2, no. 4, pp. 303-314, 1989.

[44] T. Hastie, R. Tibshirani, and J. Friedman, The Elements of Statistical Learning: Data Mining, Inference, and Prediction, Springer, Berlin, Germany, 2009.

[45] R. Hecht-Nielsen, "Theory of the backpropagation neural network," in Neural Networks for Perception, pp. 65-93, Elsevier, Amsterdam, Netherlands, 1992.

[46] A. Weigend, "Predicting sunspots and exchange rates with connectionist networks," Nonlinear Modelling Forecasting, pp. 395-432, 1992.

[47] X. Zhang, X. Chen, L. Yao, C. Ge, and M. Dong, "Deep neural network hyperparameter optimization with orthogonal array tuning," in International Conference on Neural Information Processing, Springer, Berlin, Germany, 2019.
[48] T. Yu and H. Zhu, "Hyper-parameter optimization: a review of algorithms and applications," 2020, http://arxiv.org/abs/ 2003.05689.

[49] R. Hecht-Nielsen, Kolmogorov's Mapping Neural Network Existence Theorem, IEEE Press, New York, NY, USA, 1957. 